\title{
La trilogie achevée : les sources du premier cours de linguistique générale professé par Ferdinand de Saussure
}

\author{
François Vincent $^{1}$ \\ UPEC Université Paris-Est Créteil,
}

Résumé : A Genève, Ferdinand de Saussure a professé, en élaborant progressivement son exposé, trois cours de linguistique générale répartis entre 1907 et 1912. Après en avoir examiné les notions fondamentales en suivant, entre autres cotextes, certaines des notes de lecture du maître, nous sommes revenus sur les sources et l'élaboration du premier cours. Louis Caille et Albert Riedlinger sont parmi les élèves qui ont bénéficié de ce cours, sans doute de deux façons différentes. Après avoir complété la transcription du sténogramme Caille, et effectué la transcription d'un manuscrit intermédiaire mis au jour en aout 2011, on peut comparer désormais ces deux documents avec les cahiers Riedlinger déjà connus. Ainsi préparés, ces variantes sont replacées les unes par rapport aux autres, et selon leurs natures exactes, au-delà de l'ordre chronologique de leurs découvertes respectives. La triple transcription qui en résulte - présentée selon les indices des articles de la remarquable édition de Rudolf Engler - permet de préciser les conditions d'élaboration du texte du Cours I, de le distinguer précisément du cours II et du cours III, pour pouvoir en suivre l'évolution jusqu'au fameux Cours de Linguistique Générale. Il en résulte que, de facto, la seule source à proprement parler du Cours I est ce sténogramme de louis Caille d'un part, et qu'il n'en existe très probablement pas d'autre, d'autre part. La distinction précise des éléments de la synthèse des trois cours autorise désormais l'analyse détaillée et complète du CLG publié par Ch. Bally et A. Séchehaye.

\begin{abstract}
The completed trilogy : the sources of the first general linguistics course taught by Ferdinand de Saussure. In Geneva, Ferdinand de Saussure professed, by developing gradually his presentation, three lectures of general linguistics between 1907 and 1911. Having examined the fundamental notions by following, among others texts, reading notes of the teacher, the author focused on sources and elaboration of the first lecture. Louis Caille and Albert Riedlinger are among the students who benefited from this lecture. The author completes the transcription of the stenography by Caille, and makes the transcription of a new manuscript discorvered in august 2011, and therefore can compare with the Riedlinger's already known notetakings. The triple transcription - detailed according to the ranking of the articles of the remarkable Engler's edition - allows to specify the conditions of elaboration of the text of Cours I. Besides the examination of manuscripts, of the Caille manuscript, and of its marginal notes, additional researches are made, as well on the historical conditions - genealogical and administrative concerning the registered and examined candidates -, that on the archives of the university concerning this period for the essential checks. All these informations allow to favor the hypothesis that Louis Caille acted as a transcriber for this new lecture whose introduction also modified several programs of this university. As a result, the only source strictly speaking of Cours I is this Caille stenography, contrary to what suggested until now the knowledge of texts considered inevitably in the historic order of their acquisitions; the elements of the synthesis of the three lectures - the Cours de Linguistique Générale (CLG), published by Ch. Bally and A. Séchehaye - are distinguished from each other, and its elaboration is analyzable accurately.
\end{abstract}

\footnotetext{
${ }^{1}$ fvincent66@gmail.com
} 
Deux nouveaux textes sont mis au jour et transcrits permettent de compléter les sources du CLG (Cours de Linguistique générale). Le troisième, déjà connus par son contenu, a été aussi reprit afin de permettre de comprendre sa construction ainsi que ses particularités (notes additionnelles, lacune), car c'est la mise au propre de ce texte (faite par A. Riedlinger lui-même) qui sera transmettre à Bally et Séchehaye. La reprise des indices de l'édition critique d'Engler offre la possibilité d'une comparaison des varias et de l'élaboration de la vulgate, selon toute la précision désirée. Nous avons donc la comparaison des trois textes, un sténogramme transcrit, un texte trouvé en 2011 écrit en commun par Riedlinger et Caille, ainsi que les cahiers noirs connus.

Considérer le Cours I comme un résumé est une simplification acceptable. En tant qu'exposé oral d'un essai théorisation à destination d'élèves, ou bien vu comme un résumé pédagogique, le Cours I ne doit pas être considéré isolément de l'œuvre entière. Les notions, aussi bien les notions générales sur le langage, que les notions concernant la description des langues sont expliquées dans le $C L G$, mais sont analysées et détaillées à l'occasion de nombreux cours de spécialité (Phonologie, Vieux-haut-allemand, sanscrit, versification, langues germaniques, grammaire comparée grec et latin, lituanien ...). La focalisation sur un document ne doit pas faire oublier que le texte est à l'œuvre ce que le sens est à la signification. Considérer que les conceptions de linguistique générale transparaissent à l'occasion les cours de spécialités que Ferdinand de Saussure offre, tant à Paris qu'à Genève, doit rester présent à l'esprit de son lecteur.

Plusieurs sources ont déjà été reprises (notamment dans la thèse de Robert Godel (1957) et dans l'édition critique (1967-1974) de Rudolf Engler en ce qui concerne le deuxième et le troisième Cours et certaines notes autographes préparatoires au cours. Concernant le Cours I, l'édition de Rudolf Engler, monumentale mais aussi inachevée, ne contient que le début de la transcription du sténogramme de l'élève Louis Caille. Encore plus récemment, d'autres notes nouvelles (sous le titre « De l'essence double du langage ») ont été publiées. On peut citer une lettre tirée des archives d'Engler (Bibliothèque de Genève) : « (...) Une publication intégrale des trois cours dans leur ordre naturel (et pour autant qu'ils concernent la linguistique générale) est prévue dans une collection de travaux et de documents que M. René Amaker et le soussigné [R. Engler] espèrent mettre sur pied. La collation du premier cours (cahiers d'Albert Riedlinger et sténogramme de Louis Caille est en cours) ».

Notre projet initial de thèse était une simple transcription du sténogramme. Son aboutissement final est une triple transcription indicée et alignée. Les deux états très différents de cette étude, entre génétique de texte et comparaison de varias, se trouvent en droite ligne de ce projet de R. Engler. Cette évolution est due à trois facteurs principaux :

1. Son propre développement : l'approfondissement de la compréhension des notions fondamentales, de la situation épistémologique des idées et des classifications induites, la découverte du travail de Rudolf Engler, l'étude complémentaire d'autres textes archives ou notes de lectures,

2. La découverte du manuscrit mis au jour par Daniele Gambarara, en aout 2011 : cette découverte du manuscrit intermédiaire - ultérieure, à peu près, à la finalisation de la transcription du sténogramme Caille - a impliqué le passage d'une transcription initiale à trois transcriptions, qui, en fin de compte, sont liées chronologiquement et logiquement. Les recherches complémentaires et les vérifications ont permis de conclure en privilégiant une conjecture.

3. Le choix de présentation : pour des raisons pratiques évidentes, il fallait présenter les trois transcriptions en parallèle et les indicer conformément à l'édition Engler. Ceci nous a amené d'abord à renoncer à livrer les détails techniques des choix de transcription du sténogramme; dès lors, seuls les résultats transcrits devenaient importants.

Il restait à éclaircir les traces du premier cours, ainsi que les conditions de son élaboration. Afin de séparer les textes relatifs au premier cours séparer de ceux relatifs aux deux autres cours et ainsi pouvoir reconstituer, analyser et critiquer précisément le travail des éditeurs du $C L G$.

Il s'agissait de déterminer qui avait écrit quoi, quand, à quelle occasion. Ne serait-ce que pour savoir qui avait bénéficié effectivement du Cours I. Des listes d'élèves inscrits avaient été établies sur la base de témoignages indirects, et présentaient des différences inexpliquées. Il nous a fallu relever dans les archives de l'université les listes des immatriculés, puis des inscrits au cours, enfin des élèves ayant passé l'examen (1ere et seconde session) de cours à option («Linguistique générale »). Une première confusion a donc été dégagée, et explique pour une bonne part pourquoi si peu de notes de cours pour le premier cours professé ont été retrouvés tandis qu'une dizaine ont été retrouves pour les deuxième et troisième cours. Cette confusion explique aussi pourquoi la recherche d'autres manuscrits éventuels n'avait pas et n'ont pas porté leurs fruits en plus d'un siècle. 
Les quelques élèves ayant choisi cette option de cours ont donc été déterminés, et cette liste restreinte explique aussi les annotations marginales constatées sur le sténogramme : ces élèves sont les auteurs de ces annotations que jusqu'ici on avait qualifié de « contaminations », c'est-à-dire considérées sans explications.

\section{Quelles sont donc les sources du Cours I ?}

La réponse à cette question est à la fois simple et complexe. Le sténogramme de Louis Caille (Ca), est une des sources déclarées dans la préface du CLG. Matériellement, il s'agit de sept cahiers portant, outre des signes sténographiques, plusieurs écritures différentes en marge. Sa transcription à nécessité l'apprentissage de la méthode de la sténographie Duployé. L'étude des documents d'Engler (manuscrits, tapuscrits, et fichiers), montre que celui-ci avait commencé cette transcription. En reconstituant les circonstances du cours, et les différents statuts des autres textes, nous avons pu en conclure que la sténographie Caille est de facto la seule source directe connaissable et maintenant connue, du Cours I.

Le second texte mis au jour, en août 2011, est un texte à quatre mains, celles de Caille et celles de Riedlinger, et correspond aussi à cette transcription. Ce travail commun précède le travail de Riedlinger sur ses notes du cours II et l'envoi d'une mise au propre à Bally et Séchehaye. Voici un extrait du sténogramme Caille :

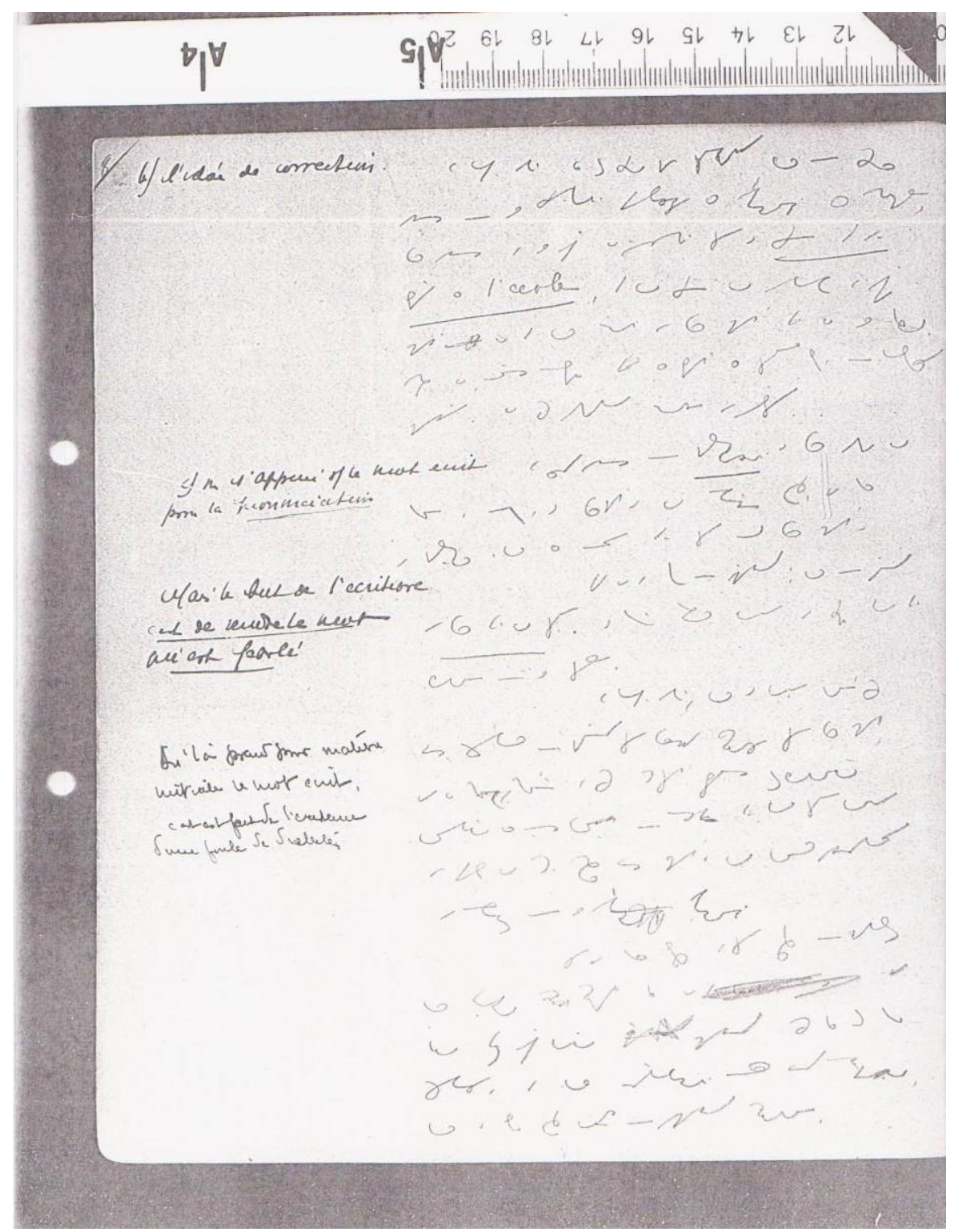


Exemple d'une page des cahiers Caille - Sténogramme et annotations marginales.

Louis Caille n'est pas et n'agit pas en sténographe professionnel : il s'agit d'un élève qui suivra le cursus de Lettres, et qui, comme beaucoup à cette époque, connait la sténographie. Malgré le caractère parfois non orthodoxe du tracé des signes sténographiques de Louis Caille, la transcription complète (de quelques 55000 signes) à été réalisée, les ambiguïtés ont été toutes discutées et tranchées. La comparaison au début du sténogramme déjà transcrit par Engler permet une vérification partielle (et ce, à quelques signes près, puisque la transcription de R. Engler n'était ni achevée ni finalisée, comme nous avons pu le montrer en examinant les différentes phases de son travail). Il est cependant possible d'apprécier le style de Caille : il ne s'agit pas d'un sténogramme au sens strict. En outre, pour quelques paragraphes de cette sténographie, on peut apprécier le style oral professoral de Ferdinand de Saussure.

Le second texte, matériellement, s'est présenté d'abord sous la forme de feuillets mélangés ayant ni le même format ni les mêmes marques imprimées. De ces différences de formats se distinguent des groupes ou blocs de feuillets, suggérant un travail discontinu, réalisé en plusieurs séances. Ces feuilles portent deux écritures au crayon, celles de Caille et Riedlinger. Après la remise en ordre de ces feuillets, le texte apparait continu, à part une lacune par rapport aux deux autres textes.

Le troisième texte, connu, est réputé être une autre source du Cours I : Les cahiers noirs dits " cahiers Riedlinger ». Il s'agit aussi de cahiers, portant de nombreuses ratures. Il n'a pas de lacune (elle est comblée), et à l'inverse, il y a des 'notes additionnelles'.

Par souci d'exhaustivité, logiquement, une tâche préalable a été de rechercher, par les méthodes de la généalogie, si parmi les descendants des inscrits au Cours I, certains avaient conservé d'autres prises de notes. Il n'y a toujours pas d'autres sources connues du Cours $I$.

Nous sommes donc en présence de trois documents et trois seulement. La triple transcription faite est présentée en tableau sur trois colonnes (plus une colonne reprenant la numérotation des paragraphes de l'édition « critique » de Rudolf Engler). Cette présentation facilite la comparaison et a fait apparaitre que nous sommes en présence de trois versions d'un même texte. On peut rajouter dans ce décompte un quatrième texte, qui n'est en fait qu'une reprise au propre des notes de Riedlinger par lui-même en vue de les transmettre aux éditeurs.

Voici un extrait de cette triple transcription :

\begin{tabular}{|c|c|c|c|}
\hline $2202 \mathrm{~d} \uparrow$ & $\begin{array}{l}\text { Dans une autre conception, c'est l'individu qui } \\
\text { prédomine. On a vu dans la langue surtout une } \\
\text { fonction parce que nous avons un organe ad hoc; } \\
\text { certains cris naturels. On observera cette fonction } \\
\text { surtout chez l'individu, mais l'individu implante à } \\
\text { la communauté la graine. }\end{array}$ & $\begin{array}{l}2^{\circ}<\text { On considère la langue surtout dans }> \\
\text { l'individu. On peut voir dans la langue surtout } \\
\text { une fonction <naturelle }>\text { (comme celle de } \\
\text { manger), parce que nous avons un appareil } \\
\text { vocal spécialement destiné à cela, cris naturels : } \\
\text { mais fonction naturelle qui ne peut s'exercer } \\
\text { qu'après avoir pris la forme de la société? }\end{array}$ & $\begin{array}{l}\text { 1Coxrigéel/Or la langue n'existe que dans des êtres } \\
\text { concrets et les collectivités ; de là, deux autres } \\
\text { conceptions : On peut voir dans la langue une } \\
\text { fonction naturelle (comme celle de manger par } \\
\text { exemple !). On considere langue surtout dans } \\
\text { l'individu : parce que nous avons un appareil vocal } \\
\text { spécialement destimé à la parole et des cris naturels. } \\
\text { Mais quelle est cette fonction naturelle qui ne peut } \\
\text { s'exercer qu'après avoir pris la forme de la société } 2[\end{array}$ \\
\hline $2202 \mathrm{e} \perp$ & $\begin{array}{l}\text { (Page 40) Une autre conception prend le côté } \\
\text { social, collectif de la langue : il s'agit non plus } \\
d^{\prime} \text { 'une fonction, mais = d'une institution sociale. } \\
\text { Mais cette institution unique est sans chair. On voit } \\
\text { que ces conceptions sont trop êtroites. }\end{array}$ & $\begin{array}{l}3^{\circ} \text { prend le langage par le côté social, collectif : } \\
\text { langue plutôt que langage <chez l'mdividu }: \text { : } \\
\text { il s'agit d'une institution sociale. C'est Mais } \\
\text { cette comme institution sociale elle est unique } \\
\text { comme fonction. Nous ne pouvons pas placer } \\
\text { donc le langage au milieu des \# choses } \\
\text { humaines. }\end{array}$ & $\begin{array}{l}\text { JInitidale2/3. idée de la langue comme } \\
\text { institution sociale. C'est la langue plutôt que le } \\
\text { langage, (qui est la langue individuel), il s'agit de } \\
\text { quelque chose de collectif, social. Cette conception } \\
\text { est plus près de la vérité que les autres, - cmais> que } \\
\text { l'on cite une autre institution comparable à celle-là : } \\
\text { la langue est un fait unique aussi bien comme } \\
\text { institution que comme fonction. [ }\end{array}$ \\
\hline
\end{tabular}

Extrait de la triple transcription : à cet endroit, les cahiers d'Albert Riedlinger contiennent trop de ratures pour qu'il soit utile de tout noter. Dans ce cas-là, unique, nous avons offert au lecteur ce qui est la première version visible et la version définitive corrigée. 
La comparaison en détail des trois textes a suscité des recherches complémentaires sur le programme de l'Université de Genève, mais encore sur les informations permettant de distinguer les immatriculés à l'Université, les inscrits au cours, les examinés aux épreuves à option. Ces informations ont permis de quantifier et d'identifier par focalisation les auteurs des différentes écritures présentes en marge du sténogramme, par-delà et en évitant la difficulté qu'aurait posé le fait de s'en tenir à la seule ressemblance graphologique.

Pour le Cours I, l'ensemble de ces données accumulées ont permis de formuler d'abord des conjectures, et d'en dégager une, plus vraisemblable et plus explicative que les autres, celle d'un Caille faisant fonction de secrétaire de séance, les trois autres élèves concernés par l'examen, annotant la marge du sténogramme lors de séances collectives de révisions, tant en première session, que pour une seconde session d'examen, pour laquelle nous avons pu montrer que Caille aide l'étudiante Whitmann, pour ses révisions d'octobre.

En outre, Riedlinger est sollicité, au moins depuis novembre 1913, par Bally pour rendre sa copie. Mais ce n'est seulement juste avant Pâques 1914 que Riedlinger prend un mois de congé pour la mise au point demandée : il aura assisté au Cours II, mais pas au Cours I, mais pourra bénéficier du concourt de louis Caille. A l'aide de cette dernière reprise du Cours I, et de la collation des Cours II et III déjà faite durant l'été par Ch. Bally et A. Séchehaye forment le CLG.

\section{Ce qui est établi : deux ordres inverses, deux visions incompatibles :}

Deux versions concurrentes et une découverte récente versus trois versions ordonnées d'une source unique. Autrement dit, un ordre historique qui est une vraisemblance - un sténogramme (Caille et « contaminations » par d'autres élèves), une prise de notes (Riedlinger), un brouillon (Caille et Riedlinger) - versus l'ordre philologique qui montre en vérité : prise de note, unique, sous forme sténographiée (Caille), première reprise lacunaire (Caille et Riedlinger), seconde reprise complétée et augmentée (Riedlinger), puis transmise.

En conséquence de quoi, de fait, la seule source à proprement parler du Cours I est le sténogramme Caille ; qu'elle restera très probablement (non pas certainement) la seule source du Cours I ; à contrario, les dits « cahiers Riedlinger $\gg$ ne constituent pas une prise de notes.

Les éléments du CLG sont identifiés, les trois cours distingués les uns des autres, et son élaboration désormais analysable complètement.

\section{Ce qui manque pour une chronologie exacte :}

Nous n'avons pas de documents prouvant positivement la période et les dates des rencontres entre L. Caille et A. Riedlinger, ni les dates de l'utilisation du sténogramme : les périodes des révisions restent indéterminées. Pour le travail de Caille et Riedlinger : le support matériel du manuscrit intermédiaire n'est pas un bloc homogène ni un ou des cahiers. Il s'agit de blocs de quelques feuillets arrachés à des cahiers ou bien des feuilles pliées sur elles-mêmes, parfois imprimées, parfois sans marges ni lignes imprimées. Cette configuration hétérogène suggère fortement plusieurs rencontres. Concernant les révisions aux deux sessions de l'examen, pour les élèves concernés, aucunes dates n'a pu être précisée ou confirmée.

Dans une lettre du 13 janvier 1914 (Archives DIP), Albert Riedlinger présente au Département de l'Instruction Publique sa demande de congé « d'un mois à compter du six ou sept janvier », comme une « requête sur le désir de Mme Ferdinand de Saussure »; le genre de la publication (œuvre de Ferdinand de Saussure) justifie la brièveté du délai avant publication, et ce, «d'autant plus que les idées saussuriennes sur la linguistique sont dans l'air » et que MM. Bally et Séchehaye « s'en rendent bien compte et voudraient, si possible, commencer à Pâques l'impression ». Il motive cette demande par l'intérêt " général et presque national de la publication », tout en proposant comme remplaçant «son ami Georges Dégallier ». Il précise enfin que la période qui nuirait le moins possible à l'enseignement de ses élèves du collège serait celle d'un mois à compter du 6 février.

Nous n'avons pas retrouvé un document démontrant la confirmation de ce congé dans les archives du DIP, ni les dates exactes accordées au professeur - nouvellement confirmé au Collège de Genève - mais ce congé semble avoir été accordé : en effet, selon une lettre (du 15 janvier 1914) à Marie de Saussure, Bally écrit s'être " assuré de la collaboration de Riedlinger, qui a demandé et obtenu un congé ». Si donc, la demande d'Albert Riedlinger lui a été accordée, on peut supposer, que cette période, à peu de jours près, se situe entre le 7 février 1914 et le 7 mars 1914 . Le jeune professeur du Collège se consacre un mois à la « mise au point » du texte du CLG et minimise ainsi sa vacance à deux semaines par rapport à ses élèves du collège. L'échéance que Bally se donne et donne à Riedlinger est donc sans doute fin mars.

La lettre du 14 mars 1914 de Bally à Marie de Saussure (BGE, Arch. de Saussure 391, f1) indique que Riedlinger a un congé pour, selon l'expression de Bally « faire le point » sur le texte du CLG. Dans une autre lettre et à propos d'un autre texte, Bally emploie cette expression « faire le point » dans le sens d'une finalisation ultime juste « avant impression », et non pour désigner une étape intermédiaire. 
Sans trace explicite plus précise, on peut donc évaluer largement la période de travail commun de Caille et Riedlinger entre la fin du Cours I et Pâques 1914 (donc après le Cours III, auquel Riedlinger n'a pas assisté), et plus strictement, probablement durant l'été 1913, été durant lequel l'essentiel de la rédaction est faite par MM Séchehaye et Bally plus ou moins alternativement, et parallèlement par Riedlinger et Caille. Son éventuelle présence au Cours I, et sa prise de note au Cours II, n'ont peut-être pas incité Riedlinger à reprendre le Cours Caille, avant la demande expresse de Ch. Bally ; mais peut-être Riedlinger a-t-il profité de l'année intermédiaire entre le Cours I et le Cours II pour travailler avec Caille... La période du travail commun entre Caille et Riedlinger n'a pas pu être déterminée plus précisément, faute de documents datés ou permettant une datation certaine. Quoi qu'ils en soient des détails historiques, toujours intéressants au demeurant, il semble que le début 1914 marque la fin de la collaboration d'Albert Riedlinger.

\section{Arguments concernant la position d'Albert Riedlinger :}

Nous avons qualifié l'immatriculation d'A. Riedlinger au Cours I de « douteuse ». Notre addendum précise ce qui suit sur la nature double des registres des inscriptions à l'Université de Genève - à la fois fiche d'inscription et documents comptables -. Nous avons repris les éléments sur la période des trois cours, sous forme numérique, ce qui permet des recoupements instantanés (en annexe de notre monographie, sous forme d'un tableau, contenant les immatriculations des quelques 512 inscriptions sur dix-sept mentions en colonnes) :

On peut constater, sur cet extrait, que pour le semestre correspondant, le registre 19 contient deux immatriculations séparées d'un jour :

\begin{tabular}{|c|c|c|c|c|c|}
\hline $17 / 01$ & & $\mathbf{9}$ & RIEDLIN & LINGUISTI & DE SAUSSURE \\
\hline 1907 & - & $\mathbf{1 8 3}$ & GER & QUE & - \\
\hline $19 / 01$ & & $\mathbf{9}^{\mathbf{9}}$ & RIEDLIN & - & GER \\
\hline 1907 & UP & $\mathbf{1 8 5}$ & & - & - \\
\hline
\end{tabular}

Extrait des registres : immatriculations d'Albert Riedlinger.

Les deux fiches sont en outre incomplètes : la première n'a pas l'indication du statut, la seconde n'a pas l'indication du cours. Les registres présentent des pages pré-numérotées (comme les livres de comptabilité), et des perforations verticales qui sont les traces de fiches détachables. La partie détachée faisait foi du paiement des droits. Ce type de format indique qu'il était sans doute prohibé ou/et impossible de rectifier une fiche, même d'un jour sur l'autre. Cette rigidité formelle fait comprendre qu'il y ait eu deux fiches distinctes (et donc deux numéros d'immatriculation), pour une seule inscription.

Cependant, en croisant la table des immatriculations et les relevés de notes des examens (repris dans les détails), on peut montrer que, pour ce qui concerne les Cours, pour chaque immatriculation qui porte la mention « sup », l'immatriculé ne passe pas l'épreuve correspondante. Cette correspondance est vraie aussi dans le cas général, pour les autres cours. On pourra noter quelques exceptions apparentes, qu'une connaissance des circonstances ou un défaut dans l'acte lui-même pourraient élucider. L'immatriculé noté " sup », auditeur ou étudiant (la fiche ne le précise alors jamais), peut ne pas vouloir, ou ne pas pouvoir, - pour toutes sortes de raisons - passer l'examen. L'immatriculé noté « sup » ne s'acquitte pas des droits d'examen : c'est ce qu'indique la mention.

Le constat déjà fait, par le décompte des semestres, en vertu duquel on a pu affirmer que Riedlinger anticipe un cours et ne peut se présenter à l'épreuve du Cours I (et même, en l'occurrence, à l'examen) est donc confirmé. L'inscription n'est pas « douteuse », elle est très peu fréquente dans les registres et sa forme requiert une explication.

\section{Conclusion}

Cette présentation insiste sur les résultats philologiques, et épistémologiques en ce sens ... Des questions comme la formalisation mathématique du système saussurien, ou bien celle d'une classification des sciences selon la nature de leurs objets respectifs, ou bien la question du problème du « mot » en tant que tel, pour une discipline qui ne peut que contenir sa propre métalinguistique ; ou bien encore la question de l'extension théorique et la position philosophique de la seconde topique saussurienne (celles des notes des « Ecrits ») sont abordées ailleurs.

Il faut conclure :

Que, s'il faut rester prudent sur la réalité historique, la recherche sur les circonstances pour établir qui avait écrit quoi et quand, et pour cela, les faits relevés - présence d'une transcription à quatre mains ; conditions effectives des cours ; des examens, des épreuves ; absence d'indice positive de l'existence d'autres prises de notes - portent à privilégier la thèse d'un Caille ayant le rôle de secrétaire de séance. Que, jusqu'à une preuve improbable du contraire, 
Louis Caille doit être considéré comme l'auteur de la seule source existante et complète du premier cours de linguistique générale.

Que par conséquences, les dits «cahiers Riedlinger » ne constituent pas une prise de notes simple du Cours $I I$ : ces cahiers noirs ont été donc improprement nommés et notés habituellement $I R$ et devraient être notés $(I-I I) R$ désormais.

Que les notions fondamentales de la pensée saussurienne sont contenues (notamment le quaternion et les deux types d'association inextricable l'une de l'autre qu'il contient) dans et dès le premier cours, et non seulement à partir du deuxième ou complètement dans le troisième, à quelques changements près dans la terminologie. Que donc, sa carrière parisienne a été aussi probablement un temps d'élaboration de concepts exposé à l'occasion de ce cours.

Qu'il ne faut pas confondre ou mélanger, c'est-à-dire qu'il faut constamment distinguer deux progressions différentes : progression de la pensée saussurienne et progression de l'exposé pédagogique saussurien.

Tous les auteurs des annotations marginales du sténogramme Caille ont été déterminés ; que ces annotations ne sont en rien des « contaminations » injustifiées. Outre ces éléments, sur les documents examinés, la nature différentielle des ajouts et des annotations faites par Albert Riedlinger montre qu'il n'est que transcripteur (de la sténographie) pour le Cours I, et qu'ensuite il produit une collation au Cours II.

Que cependant, les éléments du Cours de Linguistique Général (CLG), publié par Ch. Bally et A. Séchehaye sont distingués les uns des autres, et son élaboration est analysable en détail, selon toute la précision désirée, puisque nous avons repris, sur trois colonnes, la présentation, l'alignement, et les indices des articles de l'édition d'Engler.

\section{Bibliographie}

\section{Monographies}

- AUBENQUE Pierre, 2013, «Le problème de l'être chez Aristote » p. 222s, La science " recherchée », sciences et genre, et Chapitre II : Etres et langage, p. 94. PUF, Quadrige, 555p.

- AUBENQUE Pierre, 2009, « Problèmes aristotéliciens - Philosophie théorique », Coll. « Histoire de la philosophie », 409 pages, Antiquité Vrin.

- ARISTOTE, 2000, « Métaphysique, Nécessité d'un Premier Moteur éternel », Tome 2, Livres H-N, Traduit par Jules TRICOT, Librairie philosophique Vrin, Livre $\Lambda$, Chap. 6, p.168-172. Voir aussi : Métaphysique, Livre VI, Ch.1.

- BALLY Charles et SECHEHAYE Albert, avec la collaboration d'Albert Riedlinger, 1913, « Cours de linguistique générale » (CLG), Paris, Payot, 507p. (Exemplaire utilisé : 1996).

- BENVENISTE Emile, 1966-2004, «Problèmes de linguistique générale », pp 163-170- Tome I, Collection tel, Editions Gallimard, Paris.

- CAILLE Louis, 1906-1907, Sténographie manuscrite des notes du Cours I, BGE, Ms. Cours Univ. 762 - Ca Récemment catalogué BGE Ms. fr. 9168/4 (Antérieurement au 21/12/2012 : BGE 2010/34/3).

- CONSTANTIN Emile, 2005, « Cours III : Version du Cours selon les notes de Constantin ». Dans les Cahiers de Ferdinand de Saussure, CFS 58pp.83-289 et BGE Ms. fr. 3972.

- EGGER Victor, 1881/1904, «La Parole intérieure, Essai de psychologie descriptive », Librairie Germer Baillière, Paris.

- ENGLER Rudolf, 1967, « Ferdinand de Saussure, Cours de linguistique générale » Edition Critique (ou Philologique), Wiesbaden, Harrassowitz.

- FEHR Johannes, 2000, « Saussure entre linguistique et sémiologie », Paris PUF, 287 p.

- FOREL Claire, 2017, "Saussure, une source d'inspiration intacte », Collectif /Dir. Forel C., Editions Métis Presses, Collection Voltiges (Disponible en version numérique enrichie), Genève/FNRS (Fonds national suisse de la Recherche Scientifique).

- GODEL Robert, 1957, « Les sources manuscrites du Cours de linguistique générale de F. de Saussure », thèse éditée par la Société de publications romanes et française, Droz, Genève.

- NAVILLE Adrien, 1901, " Nouvelle classification des sciences : étude philosophique », deuxième édition, entièrement refondue, Collection Bibliothèque de philosophie contemporaine, Paris, F. Alcan, 178p.

- NODE-LANGLOIS Michel, 2008, «Petite introduction à la question de l'être : l'invention aristotélicienne de la métaphysique », Collection Questions disputées, Paris, Téqui, 135p.

- RIEDLINGER Albert (1906-1907), « Manuscrit des notes du Cours I » BGE, Ms. Cours Univ. 761 et 345 (Noté IR improprement, (I-II)R).

- SAUSSURE Ferdinand de-, 1879, « Mémoire sur système primitif des voyelles dans les langues indo-européennes », Leipsick, B. G. Teubner. Disponible sur Gallica.

- SAUSSURE Ferdinand de-, 1881, « De l'emploi du génitif absolu en sanskrit », Thèse pour le Doctorat présentée à la faculté de Philosophie de L'université de Liepzig. 
- TESTENOIRE Pierre-Yves, 2010, « Genèse d'un principe saussurien : la linéarité », Revue Recto/Verso, nº. ITEM (Institut des Textes et Manuscrits Modernes), Genèse de la pensée II : cheminements et procédures. Disponible sur hal-01395909.

- VINCENT François, 2017, Chapitre « A propos des sources du premier cours de linguistique générale. » pp 137-148. dans «SAUSSURE, Une source d'inspiration intacte », Collection Voltiges, Editeur MétisPress, Genève, 320p.

- VINCENT François, 2014, « Le premier cours de linguistique générale professé par F. de Saussure à Genève : Cours I et sténographie Caille : transcriptions et commentaires. »dans Cahiers Ferdinand De Saussure nº7, pp. 175-190. (halshs01150052v1).

- VINCENT François, 2013, « Victor Egger, Ferdinand de Saussure et la Parole Intérieure », dans Cahiers Ferdinand De Saussure n66, pp. 137-157. (halshs-01150049v1).

- VINCENT François, 2013, « Sobre a Elaboração do Primeiro Curso » - Chapitre de revue électronique, p. 78-88 in : Cadernos de historiografia linguística do CEDOCH [livro eletrônico] : Por ocasião do centenário do Curso de Linguística Geral (1916) / Cadernos de Historiografia Linguística do CEDOCH ; vol. 2), Disponible en ligne sur cedoch.fflch.usp.br/cadernos, 1. Linguística histórica. 2. Linguística (Ensino). I. Altman, Cristina, coord. II. Testa-Torelli, Lygia, coord. III. Série.

- VINCENT François, 2010, «Le premier cours de Linguistique Générale : les notes des étudiants dans l'édition et le fonds Engler. » dans Cahiers Ferdinand De Saussure n ${ }^{\circ} 63$, (halshs-01150047v1).

- VINCENT François, 2013, « Le premier cours de linguistique générale professé par Ferdinand de Saussure à Genève », Thèse de Sciences du langage, mention description des langues à l'Université Paris-Est Créteil Val de Marne, Présentée le 31 octobre (Fichier central des thèses - V0048336). L'Harmattan.

WHITNEY William Dwight, 1875, « La vie du langage »; rééd. fac-similé de l'éd. originale de 1875, Paris, Éditions

- Articles de la Revue des Cahiers Ferdinand de Saussure (Edition Droz) - (Nous utilisons la façon habituelle et

simplifiée de faire référence aux articles de l'historique revue saussurienne).

- CFS 11 (1953) GODEL Robert, La question des signes zéro, p. 31.-41

- CFS 11 (1953) GODEL Robert, texte présenté en séance, Précis de sémantique française.

- CFS 12, (1954), Note pour un livre sur la linguistique générale (1893-1894). p. 55-57.

- CFS 16 (1959) GODEL Robert, sur les listes d'élèves. p. 24s.

- CFS 16 (1959), GODEL Robert, Nouveaux documents saussuriens les cahiers E. Constantin

- CFS 17 (1960), Documents, Inventaire des documents manuscrits de F. de Saussure remis à la Bibliothèque Publique et Universitaire de Genève, p. 5-11.

- $\quad$ CFS 18 (1961), BUYSSENS Éric, Origine de la linguistique synchronique de Saussure, p. 16-33.

- CFS 21 (1964); Lettres de Ferdinand de Saussure à Antoine Meillet publiées par Emile Benveniste, p. 89-130.

- CFS 22 (1966), GODEL Robert, Mélanges de Linguistique offerts à André Burger, dans De la théorie du signe aux termes du système, p. 52-68 et Remarques sur Saussure, son système et sa terminologie, p. 35-40.

- CFS 25 (1969) GODEL Robert, Mélanges de Linguistique offerts à Henri Frei dans Questions concernant le syntagme, p.115-131.

- CFS 32 (1978) GODEL Robert, Premiers spécimens d'un index des matières au CLG/E, p. 89

- CFS 32 (1978) REDARD Georges, Deux Saussure ?, p. 27-41.

- CFS 32 (1978) HAVET Louis, Compte-rendu du Mémoire, Louis Havet et le mémoire, reprise di journal de Genève du 25 février 1879 , p. $102-122$.

- CFS 32 (1978) GODEL Robert, Les limites de l'analyse segmentales et la réalité du mot, p. 123-154.

- $\quad$ CFS 34 (1980), ENGLER Rudolf, Sémiologies saussuriennes, p.3-16.

- CFS 34 (1980), M-j. Reichler-Béguelin, dans Le consonantisme grec et latin selon F. de Saussure : le cours phonétique professé en 1909-1910, p. 27, p.17-97.

- CFS 35 (1982), GODEL Robert, Retractatio, p. 29-52.

- CFS 36 (1982), Peter Wunderli Problèmes et résultats de la recherche saussurienne, p.117-137. (CLG/E), p. 269.

CFS 42 (1988) ENGLER Rudolf, brouillon pour la note parue en 1988, Nouvelles de l'édition critique du CLG

- CFS 42 (1988) ENGLER Rudolf Diachronie : l'apport de Genève, pp. 127-166 (bilingue français-allemand) et autre article pp. 167-174 (français-italien).

- CFS 43 (1989) une Bibliographie Saussurienne 5 - 1980-1982 Travaux critiques, p. 149s.

- CFS 47 (1993-94), TURPIN Béatrice Modélisation, langage et langue chez Saussure, p. 159-175.

- CFS 49 (1995-1996) TURPIN Béatrice Discours, langue et parole dans les cours et les notes de linguistique générale de F. De Saussure, p. 251-266.

- CFS 48 (1994-1995), Correspondance Bally-Saussure, ed. Renée AMACHER, p. 91-134. Suisse, p. $134 \mathrm{~s}$

CFS 51 (1998) TRȲBA-REBER Anne-Marguerite Philologie et linguistique à l'aube du XXe siècle : l'apport de la

- CFS 51 (1998) l'erratum de la sixième Bibliographie Saussurienne 6, 1990-1997, p. 295-297.

- CFS 52 (1999) SUENAGA Akatane, Des deux arbitraires, absolu et relatif, à un arbitraire " primaire " - le fait linguistique et le devenir du signe chez Saussure, p.189-200.

282.

CFS 52 (1999) PETROFF André-Jean, La langue, l'ordre et le désordre - les analyses de Ferdinand de Saussure p. 253- 
- CFS 55 (2002) BOTA Cristian La question de l'ordre dans le cours et les écrits saussuriens de linguistique générale. Essai de refonte géométrique, p. 139-167.

- CFS 56 (2003) la Fonte di Sarna, dans un vibrant hommage se concluant sur la rigueur et l'acribie d'Engler, dans In Memoriam Rudolf Engler, p.3-18.

- $\quad$ CFS 57 (2004) STANCATI C. Saussure à l'ombre des philosophes ; p. 185-207.

- CFS 58 (2005), Version du Cours selon les notes de CONSTANTIN Emile, p. 83-289.

- CFS 58 (2005) MEJIA QUIJANO Claudia Hommage à Rudolf Engler Rudolf Engler, l'ouvrage d'un philologue artiste, p. 5-19.

- CFS 59 (2006) AMACKER René Complément à la bibliographie de Rudolf Engler, p. 218.

- CFS 63 (2010) VINCENT François -, Le premier cours de Linguistique Générale : les notes des étudiants dans l'édition et le fonds Engler, p. 229-237.

- $\quad$ CFS 66 (2013) VINCENT François Victor Egger, Ferdinand de Saussure et la Parole Intérieure, p. 137-157.

- CFS 67 (2014) VINCENT François -, Le premier cours de linguistique générale professé par F. de Saussure à Genève : Cours I et sténographie Caille : transcriptions et commentaires, p. 175-190.

Archives de l'Université de Genève

- REGISTRES des immatriculations de l'Université de Genève, dix volumes consultés. (AUG. Volumes 17 à 27).

- REGISTRE des diplômés licenciés, deux volumes. (AUG. 414A/2).

Bibliothèque universitaire de Genève

- $\quad$ ANNUAIRE 1907 de la ville Genève (BGE : Fd 351).

- BGE NC/2010/34/7. Carte de Godel à Riedlinger 15 janvier 1960, depuis Genève.

- Fonds Engler en cours de référencement contient, entre autres, les classeurs numérotés (nomenclature provisoire, définie par Daniele Gambarara, fin mai 2010, BGE) respectivement : 09.181, 17.290, 17.294 et 03.098.

- $\quad$ Fonds Albert Riedlinger. BGE 2010/34.3. Le don de M. Jean-Gabriel Riedlinger.

- $\quad$ Fond Henri Von Ziegler; BGE/BPU 1974/028.

- $\quad$ BGE/ Ms. fr. 3951

- Arch. de Saussure 372/1, f. 11 ; f15 ; f 53 ; f. 87 ; f.99 ; f176 ; f.193

- Arch. de Saussure, 372/2, f 165.

- Arch. de Saussure 372/6, f. 198

- Arch. De Saussure 372/5, f 195

- Arch. de Saussure 372/8, f 268

- Arch. de Saussure 374, f 38

- Arch. De Saussure 375/7 et 8-10 et 13 et 44v.

- Arch. de Saussure 382/9; f265 et 266.

- Arch. de Saussure 391, f1.

- Arch. De Saussure 394/1/f15.

- Cours Univ. 824, F. 219-222.

\section{$\underline{\text { Cours I }}$}

BGE Ms Cours Univ. 762 Cours Univ. (Ca ou "Cahiers Caille" ou "Sténographie CAILLE" "Cours I" ou "premier cours de linguistique générale").

- BGE Ms fr.9168/4 (« RCa », ou "manuscrit intermédiaire" ou "manuscrit à quatre mains", celles de Albert Riedlinger et Louis Caille.).

- BGE Ms Cours Univ. 761, (IR (notation usuelle mais impropre) ou plus justement (I-II)R ; ou "Cahiers noirs" ou "Cahiers Riedlinger") et Ms Cours Univ. 435, la mise au propre de ces mêmes cahiers.

Archives de la Direction de l'Instruction Publique, Genève (DIP)

- Archives DIP, 1985va 5-3-49, T2 61-6.

- $\quad$ Archives DIP, 1985va 5-3-51, Classeur 7.

- Archives DIP, 1985va 5-3-59, T2 61-6.

Sténographie

- $\quad$ A. et P. Pahud, Mme Ch. Nann, J.-F. Rouiller (1947) Cours de sténographie Duployé, manuel officiel de l'Institut Suisse, aux éditions Payot, Lausanne.

- Revue « Le Signal sténographique », Organe mensuel de l'Institut Sténographique Suisse (Fondation Duployé), Louis Mogeon fondateur, Lausanne. 